\title{
Optimal Multisine Probing Signal Design for Power System Electromechanical Mode Estimation
}

\author{
Vedran S. Perić \\ KTH Royal Institute of Technology \\ Stockholm, Sweden \\ vperic@kth.se
}

\author{
Xavier Bombois \\ Laboratoire Ampère UMR CNRS 5005 \\ Ecole Centrale de Lyon, Lyon,France \\ xavier.bombois@ec-lyon.fr
}

\author{
Luigi Vanfretti \\ KTH Royal Institute of Technology \\ Stockholm, Sweden \\ luigiv@kth.se
}

\begin{abstract}
This paper proposes a methodology for the design of a probing signal used for power system electromechanical mode estimation. Firstly, it is shown that probing mode estimation accuracy depends solely on the probing signal's power spectrum and not on a specific time-domain realization. A relationship between the probing power spectrum and the accuracy of the mode estimation is used to determine a multisine probing signal by solving an optimization problem. The objective function is defined as a weighting sum of the probing signal variance and the level of the system disturbance caused by the probing. A desired level of the mode estimation accuracy is set as a constraint. The proposed methodology is demonstrated through simulations using the KTH Nordic 32 power system model.
\end{abstract}

\section{Introduction}

Near real-time accurate monitoring of electrochemical oscillations is one of the most important functions of a wide area monitoring system (WAMS) [1]. Oscillations are monitored by continuously estimating the frequency, damping ratio and mode shape of critical system modes [2].

A traditional way of estimating modes is using an ambient system response [2]. This approach is widely accepted due to its simple implementation and the required infrastructure that does not go beyond a classical WAMS. However, the applicability of the ambient based approach is hindered by its limited accuracy, which is determined by the system's intrinsic characteristics. More accurate, but still nonintrusive, mode estimation can be performed by using a low magnitude probing signal (approximately 10 min long) as an excitation to the system [3]. In this approach, Phasor Measurement Units (PMUs) capture the system response due to the probing. As a result system identification methods that assume known input and outputs can be applied to obtain accurate mode estimates.

In addition to more accurate mode estimation, probing methods allow to identify modes that are not excited under ambient conditions, and therefore are not possible to estimate using ambient-based mode estimation.

There are several convenient ways to inject a probing signal to excite the power system:

1) By modulating the reference signal of automatic voltage regulators,

2) By modulating the reference signals of FACTS devices (active, reactive power and voltage reference signals, etc),

3) By modulating the reference signals of turbine governors.

Assuming a given location and a reference signal used for probing, there is a question on how this signal should be realized in order to obtain the best possible mode estimate. This issue was first addressed in [4] where different design considerations such as probing signal crest factor and identifiability have been discussed. In addition, [4] identifies that the frequency content (spectrum) of the probing signal is essential for the overall quality of the estimation process.

This paper investigates the relationship between the probing signal spectrum and mode estimation accuracy in order to derive a formal mathematical algorithm for optimal probing signal design. This represents a contribution in comparison with the previous methods that use probing signals that equally excite all relevant frequency components.

In the control theory community, the problem of optimal experiment (probing) design for system identification has been analyzed by numerous authors [5],[6],[7]. Recently, the least costly experiment paradigm was proposed [8],[9]. In this approach, the experiment is designed with respect to the allowed uncertainty of the estimated model. However, the focus/objective of the optimal experiment (probing) 
design has been traditionally on identification of the system's transfer function or on enhancement of the control performance, and not on the accuracy of the critical damping ratio estimate.

In this paper, the least costly paradigm is adopted for design of probing signal with the aim of guaranteeing accuracy of a few pre-defined critical damping ratio estimates, whilst relaxing requirements on accurate estimation of other model parameters. The probing signal design is formulated as an optimization problem where the decision variable is the power spectrum of the probing signal and the accuracy of the critical damping ratio estimate (described by variance of the estimate) is constrained to be lower than a pre-defined arbitrary threshold. The decision variable (i.e. spectrum of the probing signal) is parameterized (described) by the amplitudes of the sine waves in a multisine signal.

The objective function in the formulated optimization method is defined as a weighted sum of two components:

- variance of the injected probing signal that represents the probing power, and

- mean square of the output signal deviation that represents level of the system disturbance (probing impact on the system). The constraint is defined as the maximum tolerable variance of the critical damping ratio estimate.

The optimal probing power spectrum is realized using the method proposed in [4].

The reminder of the paper is organized as follows: Section 2 provides a background and underlying modeling assumptions used in deriving the method. Section 3 presents the proposed methodology, whereas the performed case studies are presented in Section 4. The main conclusions of the paper are drawn in Section 5. A list of references and an Appendix that complements the method's derivation are given in Section 6 and Section 7, respectively.

\section{Background}

During probing experiments, it is assumed that a power system can be accurately described by a linear model due to absence of large disturbances. Exploiting the system's linearity and the superposition principle, an arbitrary measured synchrophasor signal $y(t)$ can be decomposed into two components:

- A component that is as a result of ambient excitation, i.e. random load changes. This component is equal to $(H(\mathrm{z}) e(t))$, where $H(\mathrm{z})$ is a transfer function that describes aggregated behavior of the system and $e(t)$ is a white noise process that represents ambient excitation.

- A component that is a result of probing signal. This component is equal to $G(z) u(t)$. $G(z)$ represents a transfer function between probing signal and the measured output signal $y(t)$. Signal $u(t)$ is the probing signal (a disturbance introduced by a FACTS device or generator governor) that can be arbitrarily chosen by the user i.e. it can be designed with the aim to improve estimation accuracy.

The model of power system used is shown in Fig. 1:

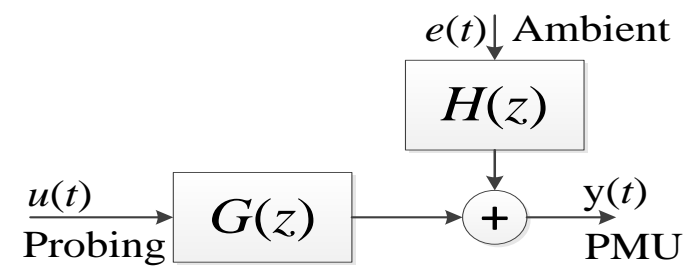

Fig. 1 Power system model during probing tests

Since both transfer functions in Fig. 1 (G(z) and $H(z))$ can be derived from the same state space model of a power system, it is reasonable to assume that both transfer functions have the same denominators. This defines an ARMAX (AutoRegressive Moving Average with eXogenous inputs) model structure of the system [10]:

$$
\begin{aligned}
y(t) & =\mathrm{G}(z) u(t)+H(z) e(t)= \\
& =\frac{B(z)}{A(z)} u(t)+\frac{C(z)}{A(z)} e(t),
\end{aligned}
$$

where $A(z), B(z)$ and $C(z)$ are polynomial functions in $z$. In addition, $A(z)$ represents the characteristic equation of the system. Note that the discrete domain is used in the model description because of the discrete nature of the measured synchrophasor signals. Also note that $H(z)$ and $G(z)$ can be parameterized (fully described) with a parameter vector $\rho$ that, among other parameters, contains the critical modes' damping ratios.

The mode estimation procedure estimates model parameter vector $\rho$ through an optimization procedure that minimizes the prediction error criterion of the model, as described in [11]. It can be shown that the covariance matrix of the parameter estimates $P_{\rho}$, in case of prediction error system identification, is given by [11]: 


$$
\begin{aligned}
& P_{\rho}^{-1}=\left(\frac{N}{\sigma^{2}} \frac{1}{2 \pi} \int_{-\pi}^{\pi} F_{u}\left(\omega, \rho_{0}\right) F_{u}^{*}\left(\omega, \rho_{0}\right) \Phi_{u}(\omega) d \omega\right)+ \\
& +\left(\frac{N}{2 \pi} \int_{-\pi}^{\pi} F_{e}\left(\omega, \rho_{0}\right) F_{e}^{*}\left(\omega, \rho_{0}\right) d \omega\right)
\end{aligned}
$$

where:

- $N$ is the number of data points used for identification,

- $\rho_{0}$ represents the true system parameter vector,

- $\Phi_{\mathrm{u}}(\omega)$ is the power spectrum of the probing signal,

- $\sigma^{2}$ is the variance of the ambient (driving) noise,

- functions $F_{u}\left(\omega, \rho_{0}\right)$ and $F_{e}\left(\omega, \rho_{0}\right)$ are defined as:

$$
\begin{aligned}
& F_{u}\left(\omega, \rho_{0}\right)=\left.H^{-1}(\omega, \rho) \frac{\partial G(\omega, \rho)}{\partial \rho}\right|_{\rho=\rho_{0}} \text {; and } \\
& F_{e}\left(\omega, \rho_{0}\right)=\left.H^{-1}(\omega, \rho) \frac{\partial H(\omega, \rho)}{\partial \rho}\right|_{\rho=\rho_{0}} .
\end{aligned}
$$

Equation (2) describes the relationship between power spectrum of the probing signal $\left(\Phi_{\mathrm{u}}(\omega)\right)$ and the accuracy of the estimate that is described by $P_{\rho}$. Furthermore, by observing (2) it is obvious that the stronger probing is, the more accurate mode estimate is obtained ( $P_{\rho}$ is smaller). Therefore, using this relationship it is possible to influence the estimate accuracy by designing the probing signal $u(t)$. This is done through an optimization process where the spectrum of $u(t)$ is a decision variable. The optimization method is described in Section 3. It has to be noted that it is assumed that $F_{u}\left(\omega, \rho_{0}\right)$ and $F_{e}\left(\omega, \rho_{0}\right)$ are known, which is typically not true. Therefore, these transfer functions have to be calculated or estimated either from the existing model of power system (using any power system modeling software), or from the initial data-based identification of $\rho_{0}{ }^{1}$. Note that $H\left(z, \rho_{0}\right)$ and $G\left(z, \rho_{0}\right)$, as well as $F_{u}\left(\omega, \rho_{0}\right)$ and $F_{e}\left(\omega, \rho_{0}\right)$, are fully described by the parameter vector $\rho_{0}$. The initial estimate of $\rho_{0}$ is a rough description of the power system behavior but sufficiently accurate to be used for design of a probing signal. It will be shown in the sequel that the identification, where the probing signal is designed in

\footnotetext{
${ }^{1}$ Initial identification of the parameter vector $\rho_{0}$ can be performed using white noise probing (or any other probing excitation that sufficiently excite the system). Also note that initial identification be done either on real system or using simulated data from the existing power system model.
}

this way, will lead to more accurate estimate of modes.

\section{Optimal Power Spectrum of the Probing Signal}

\section{A. Objective Function}

In addition to ensuring accurate mode estimation it is important to design a probing experiment (signal) that does not jeopardize system stability. In other words, the disturbance that is caused by the probing should be as small as possible. In addition, the device that is used to inject the probing signal should not be overly strained, i.e. there is a rationale to keep the power of the injected signal as small as possible. These two considerations constitute the proposed objective function of the optimization procedure that minimizes the system disturbance induced by the probing signal as well as the control effort of the probing device. This objective function, with the power spectrum of the probing signal as a decision variable, can be formalized as follows:

$$
\min _{\Phi_{u}(\omega)} J=\left(\frac{k_{1}}{2 \pi} \int_{-\pi}^{\pi} \Phi_{u}(\omega) d \omega\right)+\left(\frac{k_{2}}{2 \pi} \int_{-\pi}^{\pi}|G|^{2} \Phi_{u}(\omega) d \omega\right)
$$

where $\Phi_{u}(\omega)$ is power spectrum of the input (probing) signal and $k_{1}$ and $k_{2}$ are weighting factors. The objective function defined in this way has two summands. The first summand represents energy of the probing signal (represent strain of the probing device) and the second one represents the deviation in the energy of the selected output signal due to probing (for example frequency deviation) that reflects the level of the overall system disturbance caused by the probing experiment.

It was mentioned before that a probing signal is chosen to be a multisine that is in general case defined by the following expression:

$$
u(t)=\sum_{r=1}^{M} A_{r} \cos \left(\omega_{r} t+\varphi_{r}\right),
$$

where $A_{r}, \omega_{r}$ and $\varphi_{r}$ are the amplitude, frequency and phase of the $r$-th sine component. Consequently, the power spectrum of a multisine signal is equal to:

$\Phi_{u}(\omega)=\frac{\pi}{2} \sum_{r=1}^{M} A_{r}^{2} \delta\left(\omega-\omega_{r}\right)+A_{r}^{2} \delta\left(\omega+\omega_{r}\right)$ 
Note that the defined decision variable (power spectrum of the probing signal) is described by the coefficients $A_{r}^{2}(r=1, \ldots, M)$.

Using the defined multisine parameterization, the objective function (3) can be rewritten as follows (by substituting (5) into (3)):

$$
\min _{A_{r}^{2}(r=1 \ldots M)} J=\left(\frac{k_{1}}{2} \sum_{r=1}^{M} A_{r}^{2}\right)+\left(\frac{k_{2}}{2} \sum_{r=1}^{M}\left|G_{0}\left(\omega_{r}, \rho_{0}\right)\right|^{2} A_{r}^{2}\right),(0)
$$

Further, the relationship between the probing signal's power spectrum and the estimation covariance matrix (defined by (2)) can be expressed in terms of the multisine parameterization (by substituting (5) into (3)):

$$
\begin{aligned}
& P_{\rho}^{-1}=\frac{N}{2 \sigma^{2}} \sum_{r=1}^{M} \operatorname{Re}\left\{F_{u}\left(\omega_{r}, \rho_{0}\right) F_{u}^{*}\left(\omega_{r}, \rho_{0}\right)\right\} A_{r}^{2}+ \\
& +\frac{N}{2 \pi} \int_{-\pi}^{\pi} F_{e}\left(\omega, \rho_{0}\right) F_{e}^{*}\left(\omega, \rho_{0}\right) d \omega .
\end{aligned}
$$

Note that this expression can be simplified as:

$$
P_{\rho}^{-1}=\frac{N}{\sigma^{2}} \sum_{r=1}^{M} R_{r} A_{r}^{2}+N S,
$$

where $R_{r}$ is derived from (7), and the procedure for the derivation of $S$ given in the Appendix.

\section{B. Constraints used in the LMI optimization problem}

The accuracy of the mode estimation procedure is determined by the estimation variances of the critical modes' damping ratios $\left(\zeta_{i}\right.$.). These variances are the diagonal elements of $P_{\rho}$ (see (7)). Therefore, a sufficiently accurate mode estimate is obtained if the variance of each critical damping ratio $\zeta_{j}$ is constrained to be smaller than a desired (userdefined) threshold. This is formulated as:

$$
\begin{aligned}
& \operatorname{var}\left(\zeta_{j}\right)=P_{\rho}(i, i)=e_{i}^{T} P_{\rho} e_{i}<r \text {, i.e. } \\
& r-e_{i}^{T} P_{\rho} e_{i}>0
\end{aligned}
$$

where $r$ is the user-defined constraint (maximal allowed value of the critical variance), $i$ is the index of a critical mode's damping ratio in the parameter vector $\rho_{0}$, and $e_{i}$ is a unity vector whose $i$-th element is equal to one. When several modes need to be accurately estimated, a constraint defined by (9) is added for each critical mode.
As it can be seen from (7), the relationship between $P_{\rho}$ (or $e_{i}^{T} P_{\rho} e_{i}$ ) and the decision variable (power spectrum of the probing signal) is non-linear due to the inversion operation. In order to formulate an LMI form of the constraint defined by (9), this relationship has to be convexified. This can be done by exploiting the Schur complement property that a matrix is positive definite if and only if its Schur complement is positive definite [12]. As (9) can be represented in the form of Schur complement, an equivalent constraint would be a constraint on the positive-definiteness of the matrix whose Schur complement is equal to $r-e_{i}^{T} P_{\rho} e_{i}$. Therefore, the constraint (9) can be written as:

$$
\left[\begin{array}{cc}
r & e_{i}^{T} \\
e_{i} & P_{\rho}^{-1}
\end{array}\right]>0 .
$$

The constraint defined by (10) has a form of an LMI. This can be seen when $P_{\rho}^{-1}$ in (10) is replaced by (8).

Another constraint that has to be taken into account is that the obtained power spectrum must be positive for all frequencies (physical constraint). The spectrum's positivity is guaranteed by imposing nonnegativity of the decision variable, as follows:

$$
A_{r}^{2} \geq 0 ; \quad \text { for } r=1,2, \ldots, M .
$$

The discussion above defines an optimization problem with its objective function (power of the probing signal and the level of disturbance induced by the probing signal), decision variable (spectrum of the probing signal) and constraints (desired accuracy of the estimate defined by the maximum allowed variance of the critical damping ratio). The solution of this optimization problem provides a spectrum of the probing signal that results with a) the desired accuracy and b) minimal impact on the normal power system operation.

For the sake of simplicity, the defined optimization problem is summarized and rewritten in the sequel:

\section{Objective:}

$$
\min _{A_{r}^{2}(r=1 . . M)} J=\left(\frac{k_{1}}{2} \sum_{r=1}^{M} A_{r}^{2}\right)+\left(\frac{k_{2}}{2} \sum_{r=1}^{M}\left|G_{0}\left(\omega_{r}, \rho_{0}\right)\right|^{2} A_{r}^{2}\right)
$$

subject to: 


$$
\begin{aligned}
& {\left[\begin{array}{cc}
r & e_{i} \\
e_{i}^{T} & \frac{N}{\sigma^{2}} \sum_{r=1}^{M} R_{r} A_{r}^{2}+N S
\end{array}\right]>0,} \\
& A_{r}^{2} \geq 0 ; \quad \text { for } r=1,2, \ldots, M,
\end{aligned}
$$

where all variables are defined before.

Once the power spectrum has been determined it is necessary to realize the time-domain representation of the probing signal that will be added to a reference signal fed to a controller of a probing device. It is known that one signal spectrum can be represented with different time-domain representations. Therefore, it possible to choose the one that fits the best according to other (secondary) criteria that can be introduced in order to improve the overall estimation process. In the methodology proposed in [4], the crest factor is used as a signal quality indicator and the time-domain signal realization process is used for further optimization, where the crest factor is minimized. This time-domain probing signal realization methodology from [4] is applied here without any alterations.

\section{Case Studies}

The case studies are performed using the KTH Nordic 32 test system shown in Fig. 2 [13]. It is assumed that a FACTS device with the capability of injecting reactive power is installed at bus 48 and used for probing. As an output, the voltage magnitude of bus number 38 is selected. The disturbance is evaluated using the same output signal, i.e. the deviation of the voltage magnitude is selected as a measure of the disturbance caused by the probing procedure. The KTH Nordic 32 test system has two critical modes, one at $0.5 \mathrm{~Hz}$ and another at $0.76 \mathrm{~Hz}$. The probing signal is designed to accurately estimate the damping ratios of these two modes.

It was mentioned that an initial estimate of $\rho_{0}$ is required to perform optimal probing signal design. This initial estimate is obtained through an identification procedure where the data are generated with the linearized high-order power system model [13]. The ambient excitation (active and reactive power injections) is modeled by unity variance white noise in all load buses, whereas the input (probing) signal is chosen to be white noise with a variance of 10000 . The order of the identified models $(G(z)$ and $H(z))$ are chosen to be equal to 12 . This initial estimate has a limited accuracy that will be improved by using the optimal probing. The duration of the desired optimal probing signals is chosen to be 10 minutes, i.e. 3000 data samples (sampling frequency is equal to $5 \mathrm{~Hz}$ ).

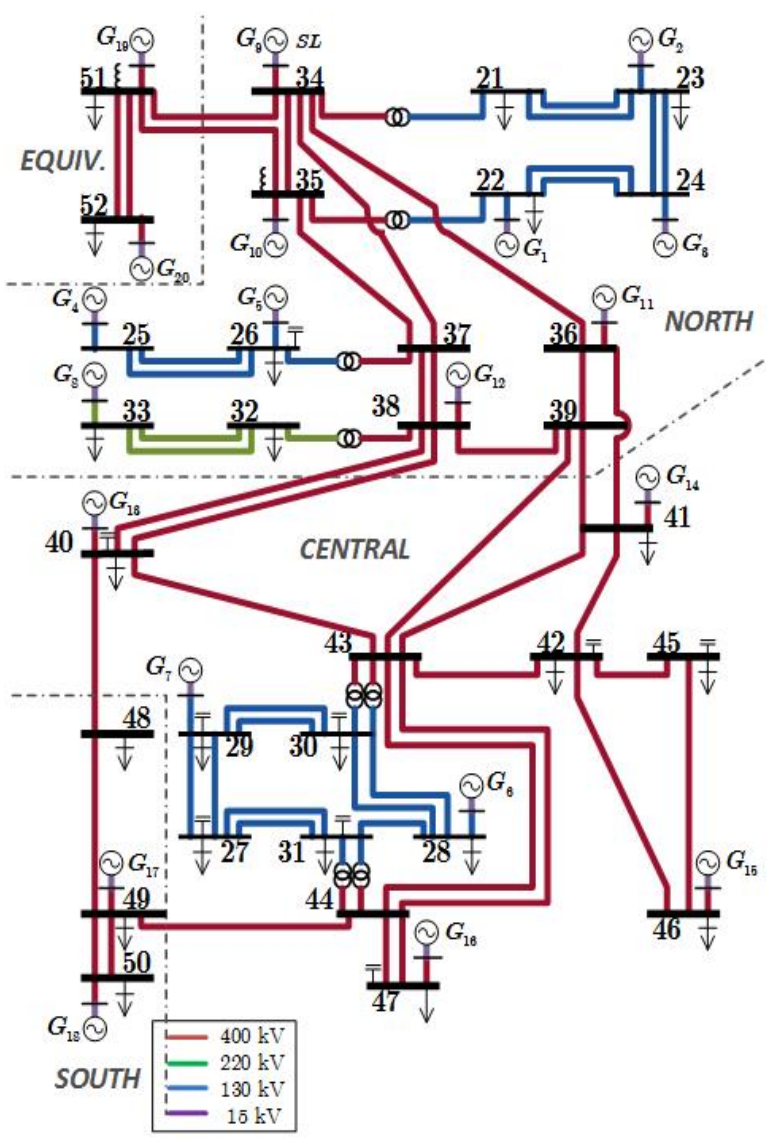

Fig. 2 KTH Nordic 32 test system

\section{Optimal power spectrum computation}

In the first case study, the power spectrum of the probing signal is computed with the objective to minimize its variance $\left(k_{1} \neq 0\right.$ and $\left.k_{2}=0\right)$. The variance of the damping ratios' estimates is constrained to be smaller than $10^{-5}$ (for both modes that corresponds to $0,361 \%$ standard deviation of the damping ratio estimate). In the following case studies, the probing power spectrum is designed as:

1) white noise,

2) multisine.

The variance of the white noise probing signal is obtained directly from (3) by replacing $\Phi_{u}(\omega)$ with a constant function (with the desired mode estimate accuracy). The multisine signal is designed with a frequency resolution of $0.01 \mathrm{~Hz}$ and components from $0.05 \mathrm{~Hz}$ up to $2.5 \mathrm{~Hz}$.

The probing spectrum obtained is shown in Fig. 3. 


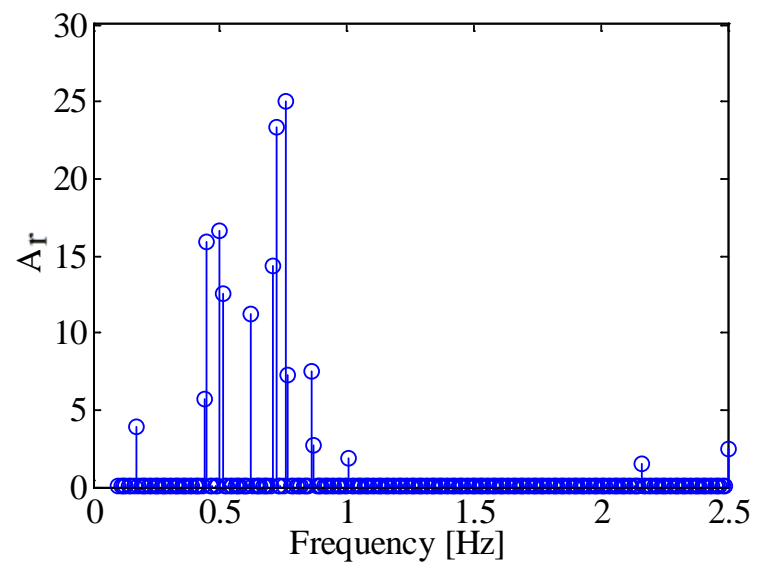

Fig. 3. Spectrum of the optimal multisine input signal when input variance is minimized

The signal's energy is mostly allocated around the critical modes' frequencies $(0.5 \mathrm{~Hz}$ and $0.76 \mathrm{~Hz})$. This is understandable because the easiest way to excite the mode is to excite exactly frequency of the mode (this is the frequency where the system has the highest gain). However, this also may introduce a risk for the system's stability because potentially a latent low damped/unstable mode could be excited.

Next, as a second case study, the objective is to minimize the disturbance introduced by the probing signal $\left(k_{1}=0\right.$ and $\left.k_{2} \neq 0\right)$. The obtained probing spectrum obtained is shown in Fig. 4.

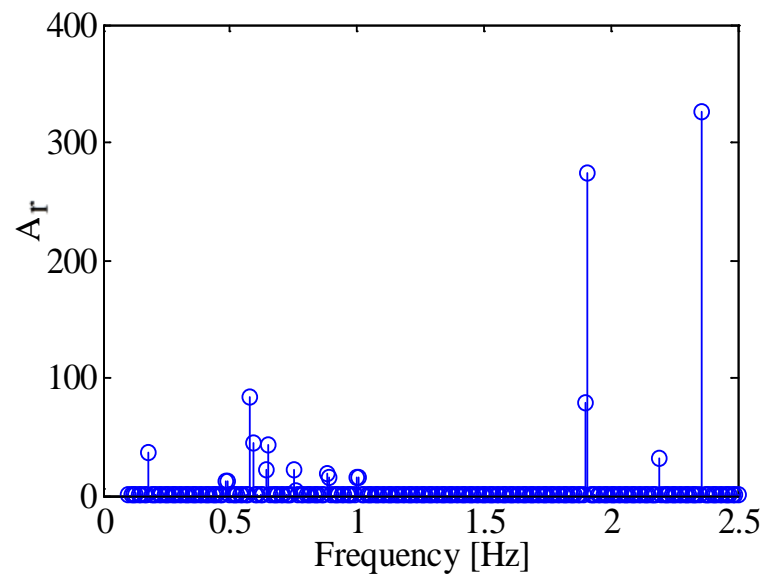

Fig. 4. Spectrum of the optimal multisine probing signal when output variance is minimized

It can be seen that the variance of the probing signal is much larger as compared to the case when the probing signal's variance was minimized. It is interesting to note that high frequency components carry most of the signal's power. This is because of the low system gain at these frequencies; therefore a probing signal with such components has a negligible impact on the system's response. In other words, the method avoids exciting frequencies that are close to the critical mode and tries to capture enough information about the system's model by exciting high frequency components ${ }^{2}$. This is a desirable property because it might be dangerous to excite the exact frequency of the modes, as explained in the previous study case.

In order to minimize both probing (input) and output variance, a weighted sum is taken as a criterion (denoted by $\operatorname{var}\{u y(t)\})$. The weighting factors in (6) are chosen to have the following values: $k_{1}=0.5$ and $k_{2}=1000$, because the output variance has a numerical value that is roughly 2000 times smaller. The obtained probing spectrum is shown Fig. 5.

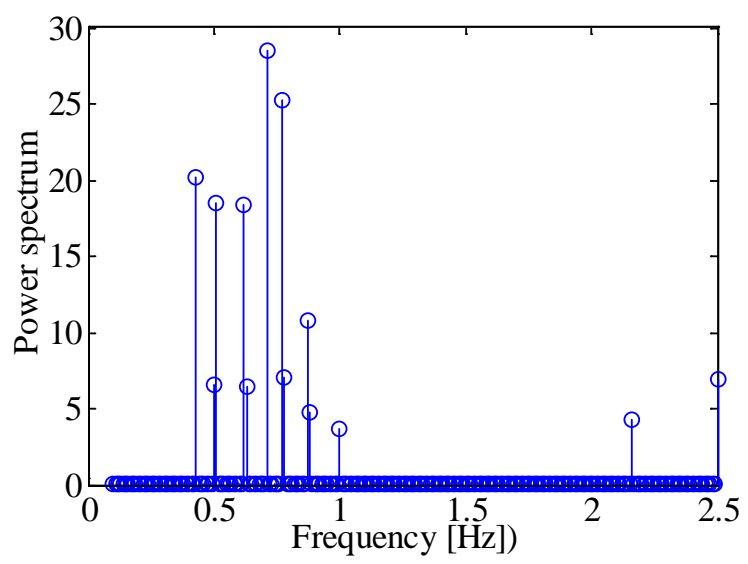

Fig. 5. Spectrum of the optimal multisine probing signal when both, probing and output variances are minimized

This type of criterion represents a compromise between the two previously presented criteria (input only and output only criteria). It can be noted that high frequencies do not contribute significantly to the accuracy of the estimated modes, and thus, as shown in Fig. 5, these components are suppressed (comparing to the case when only output variance is minimized, see Fig. 4). The result is that the power spectrum's shape is similar to the case when only the probing signal's variance is minimized (the energy of the signal is mostly allocated around the critical modes' frequencies, see Fig. 3). The numerical

\footnotetext{
${ }^{2}$ As the system has small gain at high frequencies, the cost in the objective function that corresponds to these frequencies is small as well, regardless of the strong excitation by probing signal (the objective function is proportional to the product of system gain and input power at each particular frequency)
} 
values of the probing signal obtained and output signal variances are shown in Table I.

Table I Introduced system disturbance when the variance of the input and output signal is minimized.

\begin{tabular}{llccc}
\hline \hline & White & \multicolumn{3}{c}{ Minimized function } \\
& noise & $\operatorname{var}\{u(t)\}$ & $\operatorname{var}\{y(t)\}$ & $\operatorname{var}\{u y(t)\}$ \\
\hline $\operatorname{var}\{u(t)\}$ & 10410 & 1179.8 & 101850 & 1441.6 \\
$\operatorname{var}\{y(t)\}$ & 1.6761 & 2.0915 & 1.2425 & 1.5980 \\
$\operatorname{var}\{u y(t)\}$ & 6881.1 & 7981.4 & 52167 & 2318.8 \\
\hline
\end{tabular}

Table I summarizes the most important results. The reference case represents the situation when probing is performed using white noise signal (spectrum is not optimized, rather all frequencies are equally excited). It can be seen that when the input (probing) power is minimized, the same mode estimation accuracy can be accomplished with an input power that is roughly 8 times smaller (10410 compared to 1179.8). This is a significant improvement when the capacity of injecting disturbance is limited. However this type of probing may pose a risk for power system stability because the output variance (that represents a level of system disturbance) is the largest in this case (2.0915)

On the other hand, when only the system disturbance is minimized (arbitrary power level of probing signal is allowed), a significantly larger probing power (by factor of 90) is obtained to accomplish the same level of desired mode estimation accuracy. This level of probing input power might be unacceptable from the perspective of the probing device's allowable strain, so a tradeoff between these two extreme cases (as presented by the case where both input and output powers are minimized) might be the most suitable for real-life applications.

It can be also noticed that the space for improvement in terms of output power (level of system disturbance) is generally much smaller than the space for improvement of the level of input power. This is reasonable, because a desired mode estimation accuracy inevitably implies a significant level of system disturbance (if the system is not sufficiently disturbed, no useful information about the modes can be extracted from the measured outputs).

At this point it is important to clarify what the numbers in Table I represent quantitatively. Because a linearized model was used, the level of excitation (ambient noise) does not directly affect the shape of optimal spectrum result (linearity property). What is important is the variance ratio between the ambient noise and the probing signal. In the studies presented, the ambient noise is represented by a unit variance white noise active and reactive power injections at all buses of the linearized model. This means that the reactive power probing of 10410 (Table I) corresponds to a value that has $\sqrt{10410} \approx 100$ larger magnitude in per unit than the ambient noise (which is assumed to have unity variance). This further means that an ambient excitation of 100kVA at each bus requires a probing signal of 10MVA for the presented case. Because of the variable nature of the ambient noise, it has been decided to present the relative values shown in Table I rather than absolute values.

\section{Signal realization}

As presented in [4] it is beneficial when the probing signal has a time-domain representation with a small crest factor. In the case of multisine signals, this means that the vector of sine waves' phases has to be optimized with the objective of minimizing crest factor. This minimization procedure is given in [4]. Table II presents the values of the crest factors for signals whose spectra where determined earlier.

It can be noticed that the obtained crest factors are reduced significantly, as compared to the case with random phases of sine components. However, the values depend on the spectrum and phases used for initialization.

Table II Crest factors of the designed multisine signals.

\begin{tabular}{llc}
\hline $\begin{array}{l}\text { Objective } \\
\text { function }\end{array}$ & \multicolumn{1}{c}{ Method } & Crest Factor \\
\hline \multirow{2}{*}{$\operatorname{var}\{u(t)\}$} & Random phases & 4.034 \\
& Optimal phases & 3.272 \\
\hline \multirow{2}{*}{$\operatorname{var}\{y(t)\}$} & Random phases & 2.863 \\
& Optimal phases & 2.618 \\
\hline \multirow{2}{*}{$\operatorname{var}\{u y(t)\}$} & Random phases & 3.579 \\
& Optimal phases & 2.754 \\
\hline
\end{tabular}

\section{Conclusion}

Probing-based mode estimation enables more accurate estimation in comparison with ambient based methods due to a known disturbance injected into system. Probing experiments in power systems are costly processes that have to be carefully planned. One of the important design considerations in the planning stage is the shape of the probing signal. This paper proposes a methodology for probing signal design that exploits the relationship between the probing signal spectrum and the accuracy of the mode estimate. It is shown that only the power 
spectrum of the signal (not the time-domain signal realization) determines the accuracy of the mode estimation process. Consequently, the spectrum of the probing signal is determined through an optimization process, where the impact of the probing on the normal system operating condition is minimized, whilst the desired mode estimation accuracy is treated as a hard constraint. The probing signal is modeled as a multisine signal where the spectrum is described by the amplitudes of the individual sine components (determined through optimization). Once the probing power spectrum has been determined (first stage), a time-domain probing signal is generated with the aim to minimize crest factor of the signal in the second stage.

The presented method provides a significant improvement in comparison with previous probing signal design methods that excite all frequency components equally. It is shown that the same accuracy of mode estimation can be accomplished with the probing signal that has roughly 8 times smaller power. In addition, it is also shown how to minimize the impact of the probing signal on the system's operating condition.

The advantage of the proposed method is its straightforward application in real-life conditions. The probing signal is determined offline and later reproduced using reference inputs of FACTS controllers or generator regulators. The presented method assumes the existence of the initial model that is used for probing design. This assumption may lead to a suboptimal signal design, which is a drawback that will be tackled in future research efforts.

\section{References}

[1] F. Galvan and P. Overholt, "The intelligent grid enters a new dimension”, $T \& D$ World, July 28, 2014.

[2] J.J. Sanchez-Gasca (Ed.), "Identification of electromechanical modes in power systems", IEEE Task Force Report, Special Publication TP462, 2012.

[3] N. Zhou, J.W. Pierre and J.F. Hauer, "Initial results in power system identification from injected probing signals using a subspace method”, IEEE Trans. Power Syst., vol.21, no.3, pp.1296-1302, Aug. 2006.

[4] J.W. Pierre, et al. "Probing signal design for power system identification”, IEEE Trans. Power Syst., vol.25, no.2, pp.835-843, May 2010.
[5] M. Gevers, X. Bombois, R. Hildebrand and G. Solari, "Optimal experiment design for open and closed-loop system identification”, Communications in Information and Systems, vol.11, no.3, pp.197-224, 2011.

[6] C.R. Rojas, J.S. Welsh, G.C. Goodwin and A. Feuer, "Robust optimal experiment design for system identification”, Automatica, vol.43, no.6, pp.993-1008, June 2007.

[7] U. Forssell and L. Ljung, "Some results on optimal experiment design”, Automatica, vol.36, no.5. pp.749-756. May 2000.

[8] X. Bombois, G. Scorletti, M. Gevers, P.M.J. Van den Hof and R. Hildebrand, "Least costly identification experiment for control”, Automatica, vol.42, no.10, pp.1651-1662, Oct. 2006.

[9] X. Bombois and G. Scorletti, "Design of least costly identification experiments", Journal Européen des Systèmes Automatisés, vol.46. no.6-7, pp.587-610, 2012.

[10]L. Dosiek and J.W. Pierre, "Estimating electromechanical modes and mode shapes using the multichannel ARMAX model”, IEEE Transactions on Power Systems, vol.28, no.2, pp.1950-1959, May 2013.

[11]L. Ljung, System Identification Theory for the User, Englewood Cliffs, N.J.: Prentice Hall, 1999.

[12]Z. Fuzhen (ed.), The Schur complement and its applications, Springer, 2005.

[13] Y. Chompoobutrgool, W. Li and L. Vanfretti, "Development and implementation of a Nordic grid model for power system small-signal and transient stability studies in a free and open source software”, in Proc. IEEE Power Eng. Soc. General Meeting 2012, 22-26 July 2012.

\section{Appendix}

This appendix presents the derivation of expression (8) that has a LMI from as defined with (7). These two expressions are rewritten here for the sake of derivation completeness:

$$
\begin{aligned}
& P_{\rho}^{-1}=\frac{N}{2 \sigma^{2}} \sum_{r=1}^{M} \operatorname{Re}\left\{F_{u}\left(\omega_{r}, \rho_{0}\right) F_{u}^{*}\left(\omega_{r}, \rho_{0}\right)\right\} A_{r}^{2}+ \\
& +\frac{N}{2 \pi} \int_{-\pi}^{\pi} F_{e}\left(\omega, \rho_{0}\right) F_{e}^{*}\left(\omega, \rho_{0}\right) d \omega . \\
& P_{\rho}^{-1}=\frac{N}{\sigma^{2}} \sum_{r=1}^{M} R_{r} A_{r}^{2}+N S
\end{aligned}
$$


As stated before, $R_{r}$ is derived trivially from $\mathrm{A}(1)$, whereas the derivation of $S$ is given in the sequel. First, it is necessary to compute the matrix $S$, that is equal to:

$$
S=\frac{1}{2 \pi} \int_{-\pi}^{\pi} F_{e}\left(\omega, \rho_{0}\right) F_{e}^{*}\left(\omega, \rho_{0}\right) d \omega .
$$

Using Parseval's theorem this expression can be rewritten as follows:

$$
\begin{aligned}
& S=\frac{1}{2 \pi} \int_{-\pi}^{\pi} F_{e}\left(\omega, \rho_{0}\right) F_{e}^{*}\left(\omega, \rho_{0}\right) d \omega= \\
& =E\left[\bar{y}(t) \bar{y}^{T}(t)\right]
\end{aligned}
$$

where $E$ denotes the expected value of a random process, $\bar{y}(t)=F_{e}(z) \bar{e}(t)$ with $\bar{e}(t)$ white noise of unit variance.

Note that the transfer function $F_{e}(\mathrm{z})$ can be expressed as a single-input multiple-output (SIMO) state space form, as follows:

$$
\begin{aligned}
& \bar{x}(t+1)=A \bar{x}(t)+B \bar{e}(t) \\
& \bar{y}(t)=C \bar{x}(t)+D \bar{e}(t) .
\end{aligned}
$$

Using (A5), the expression for transfer function $F_{e}(\mathrm{z})$ can be further manipulated as follows:

$$
\begin{aligned}
& F_{e}(\mathrm{z})=E\left[\bar{y}(t) \bar{y}^{T}(t)\right]= \\
& =E\left[(C \bar{X}(t)+D \bar{e}(t))(C \bar{x}(t)+D \bar{e}(t))^{T}\right] \\
& =E\left(C \bar{X}(t) \bar{x}^{T}(t) C^{T}\right)+E\left(D \bar{e}(t) \bar{e}^{T}(t) D^{T}\right) \\
& =C X C^{T}+D D^{T} .
\end{aligned}
$$

Equation (A9) constitutes the Lyapunov equation $X=C X C^{T}+D D^{T}$ that can be solved for $X$, providing a solution for $S$, i.e. $S=X$.

This concludes the derivation of $\mathrm{S}$ and this appendix. 